\title{
Usability Evaluation of Mobile Tracking Applications: A Systematic Review
}

https://doi.org/10.3991/ijim.v14i05.13353

\author{
Murtaja Ali Saare, Azham Bin Hussain ${ }^{(凶)}$, Osamah Mohammed Jasim \\ Universiti Utara Malaysia, Kedah, Malaysia \\ azham.h@uum.edu.my \\ Alia Ahmed Mahdi \\ Directorate of Health, Baghdad, Iraq
}

\begin{abstract}
In the recent years, mobile is found to be useful since they allow the user to perform more tasks in a mobile context. However, this usefulness come at the expense of the usability of these devices in some contexts. Thus, usability becomes an important factor in the adoption of mobile applications (such as mobile tracking applications, mobile health and mobile learning), which are often used by people who have problems when using mobile devices and who have a limited experience of technology. It is critical in the Mobile domain to ensure the success of mobile applications. Despite some studies review usability evaluation, regrettably there are no systematic literature reviews focused on usability evaluation of mobile tracking applications. Consequently, in this study, the researchers strive to present the results of a systematic literature review (SLR) involving the use of usability evaluation techniques in Mobile tracking applications. Based on three research questions, the results indicated that, there are three kinds of the Mobile tracking applications namely: information tracking, location tracking, information, and location tracking. In addition, and regard to the techniques used to evaluate the usability, the experimental study was the regularly used in the selected studies. Finally, the results shown that, for characteristics were frequently exploited to measure the usability of mobile tracking application are Effectiveness, Efficiency, Usefulness and Accurate. This study is valuable for the researchers who need to evaluate the usability for any new mobile application through explored the appropriate methods used to evaluation the usability and indicators required for this purpose.
\end{abstract}

Keywords - Usability Evaluation, Mobile Device, Tracking Application, Systematic Literature Review.

\section{$1 \quad$ Introduction}

Mobile devices are becoming gradually popular, among consumers [1], [2], and have already reached over one billion mobile subscribers [3]. The most popular mobile technology now is Smartphone. Worldwide information shows that one out of 
five people own the smartphone [4]. Smartphone is more sophisticated computing ability and connectivity as compared to mobile phones. The extended input mode is supplied with a touch sensitive display. Though in 1992, IBM had created its first touchscreen smartphone, called Simon Personal Communicator; Ericsson R380 launched in 2000 was the first mobile phone to be commercialized as a "smartphone".

The first smartphone powered by its touchscreen was released in Apple in 2007: the iPhone [5]. The Android operating system was launched in 2008 for mobile touchscreen devices. The innovation of the first iPhone has governed the development of smartphones. Android, IOS, Windows Phone and BlackBerry are the most popular mobile OS for smartphones [6]. In addition, these OSs are designed to work on touchscreens tablet computers. In recent years, too, tablets have become popular. At the end of 2013 roughly $6 \%$ of the global population had a tablet. The growth of application stores is one of the key factors leading to adoption [7] [8].

However, even if progress in technological innovations has been made, the features of mobile devices (small screen size, low display resolution, non-traditional input methods, and navigational difficulties) are clearly restrictive to and difficult to use mobile device interfaces [9]. Usability, therefore, is more essential than for other areas in mobile technology because many mobile apps still are difficult to operate, flexible and lack robust.

The usability of human-computer interaction (HCI) research is a well-known and well-defined concept which refers to the degree to which users and computers can "communicate" clearly through interfaces. Coursaris and Kim [10] also said that usability for smartphones has become an important topic because applications need to be prevented from being difficult to use; it has been recognized as one of the determinants of their success. In addition, despite significant research on overall usability, relatively little studies were conducted with regard to mobile usability due to the innovation in mobile technology [10][11], in particular using systematic review approach. There are many mobile applications currently available [12], this systematic study will focus on the new phenomenon harness mobile devices to guide the user on the right way or at the level of progress the transactions, are known as mobile tracking (or M-tracking).

\section{Systematic Literature Review Methodology}

The Systematic Literature Reviews (SLR) summarizes the research evidence[13]. According to Kitchenham[14], SLR is "a means of evaluating and interpreting all available research relevant to a particular research question or topic area or phenomenon of interest". Indeed, in this systematic study the researchers adopted the guidelines for conducted SLR based on the parameters defined by [15]. In the present study, the activities that facilitated the process of SLR includes formulation of research questions, identification of search syntax, selection of resources (e.g. journal articles), extraction of data from primary sources, and reporting the data extracted, the Fig. 1 highlights the stages of the current study. 


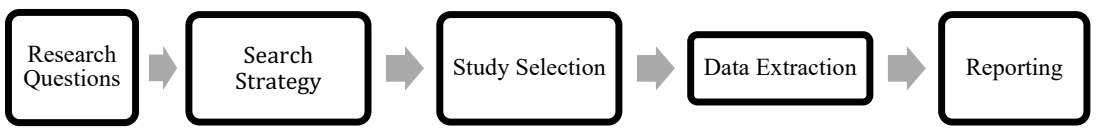

Fig. 1. Stages to conduct the systematic review method

\subsection{Research questions}

The main objective of the present study was to determine the types of the tracking applications and to explore the main usability evaluation methods used on the previous studies. In order to attain this goal, we defined a set of questions to be addressed by this review:

1. What the kinds of the mobile tracking applications has been designed?

2. How to evaluate the usability of the mobile tracking applications?

3. What are the usability evaluation characteristics used by prior studies?

\subsection{Search strategy}

To perform the search process and selection the particular studies pertaining to this study, the researchers defined research strategy that will be performed in the present study based on the two question which listed in the previous subsection.

Search terms: In this section search terms will be chosen based on the three research questions that formulated previously. Because the usability evaluation method too broad and attempt to cover all usability methods is very difficult. Nevertheless, the study defined the following basic search strings:

- C1: "Smartphone OR smart phone OR touchscreen OR mobile phone OR mobile devices OR tablet OR mobile technology"

- C2: "Tracking OR Mobile tracking OR tracking system OR mtracking"

Therefore, the complete string will be exploited in this study was:

(C1 AND C2) AND/OR (C1 OR C2)

\subsection{Search process}

In this study focused only on the open access online libraries and the digital resources which available for the students in University Utara Malaysia, namely:

1. ACM Digital Library (portal.acm.org/dl.cfm)

2. IEEE Electronic Library (ieeexplore.ieee.org)

In addition to the digital resources mentioned above, the researchers also searched in Google scholar (http://scholar.google.com.my). 


\subsection{Study selection}

A set of selection criteria were used considered while selecting appropriate literature for this SLR. Generally speaking, to determine which piece of studies were included, the researchers placed the following criterion:

1. Studies not written in English language

2. The study not relevant to present research questions

3. The study that is not within the specified time frame

4. Duplicate study in different digital resources

5. No empirical study.

Fig 2 summarizes the appropriate criteria for this study to determine the valuable and relevant studies of such systematic literature review. However, as shown in Fig 2, this study concentrated on studies published between 2006 and 2016. The reason selected this period, because during this period the mobile device witnessed a major development[16], [17].

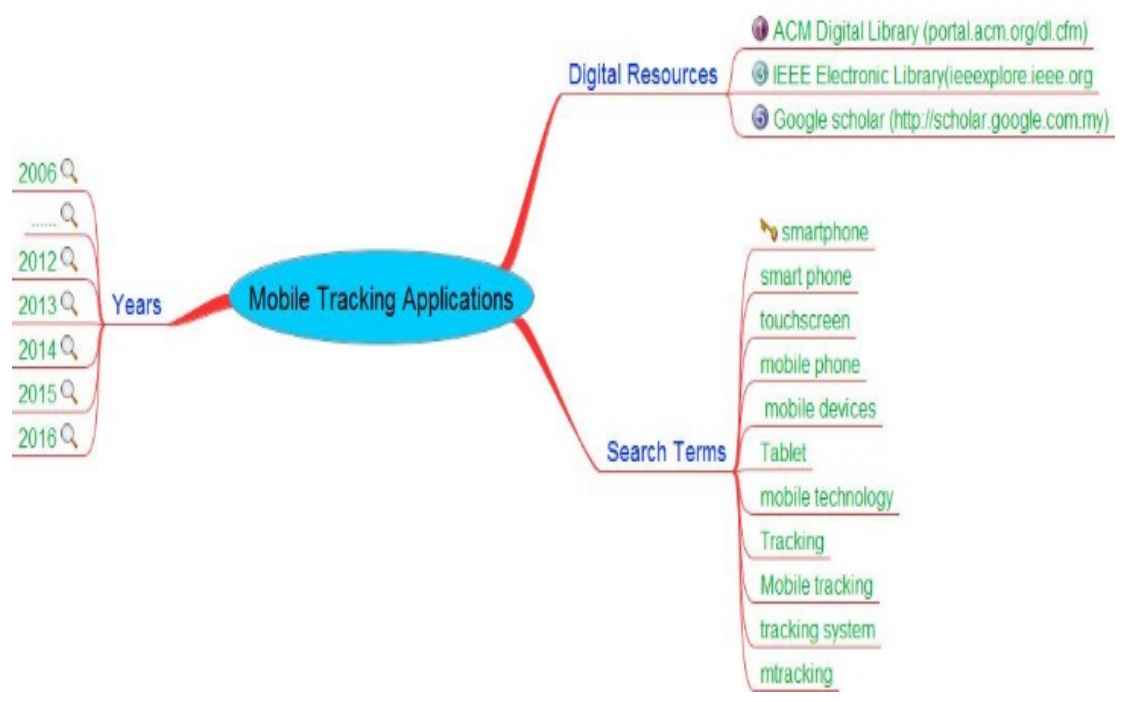

Fig. 2. Illustrate the criterion to select appropriate studies

\subsection{Data extraction}

To answer the research questions formulated above data extraction process was carried. According to Kitchenham[18]it allows the researcher to gather information required from the different studies. In most of the systematic studies, data extraction defined a set of numerical values that should be extracted for each study. The current systematic review will depend on the strategy that conducted by[15], [17]. 


\subsection{Synthesis strategy}

Data synthesis involves gathering and summarizing the results of the only included studies[17]. In the first round, 120 papers have been obtained, as shown in Fig 3. Out of the 120 papers, [15] were excluded because several issues such as duplicates, language and out-of-date. The remaining 103 papers were evaluated by considering their title and keywords. 30 papers were excluded in this round. In the third round, $40 \mathrm{pa}-$ pers were excluded after examining their abstracts. The full texts of the remaining 33 papers were investigated, 9 papers were discarded (since they were not relevant with current study) and only 24 papers were selected after applying the remaining criteria.

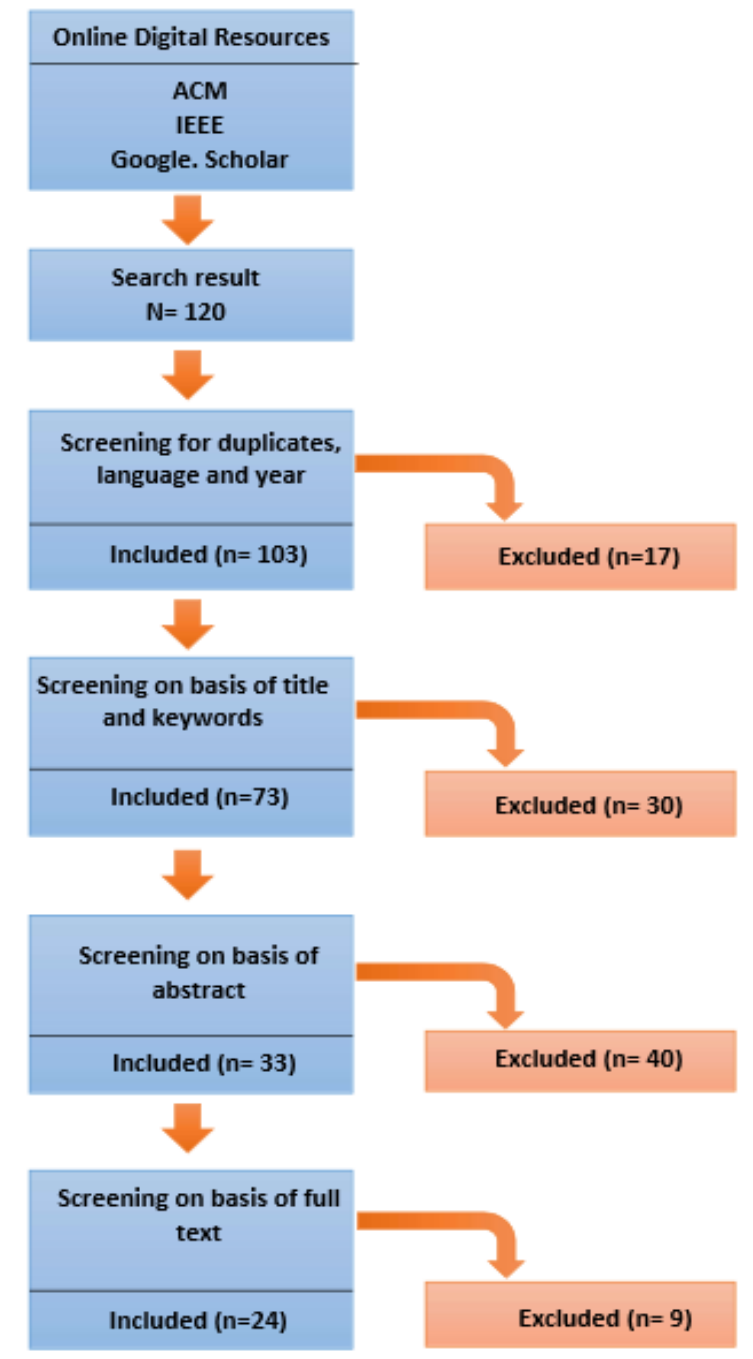

Fig. 3. PRISMA flow diagram 


\section{Results}

Three questions were established in this systematic review. With regard to the first research question, it is discovered the categories of mobile tracking applications. Second research question explored the usability evaluation methods harnessed via previous studies for assessing mobile tracking apps. Finally, identify what are the characteristics that have been used to measure these applications.

RQ1: What the kinds of the mobile tracking applications has been designed?

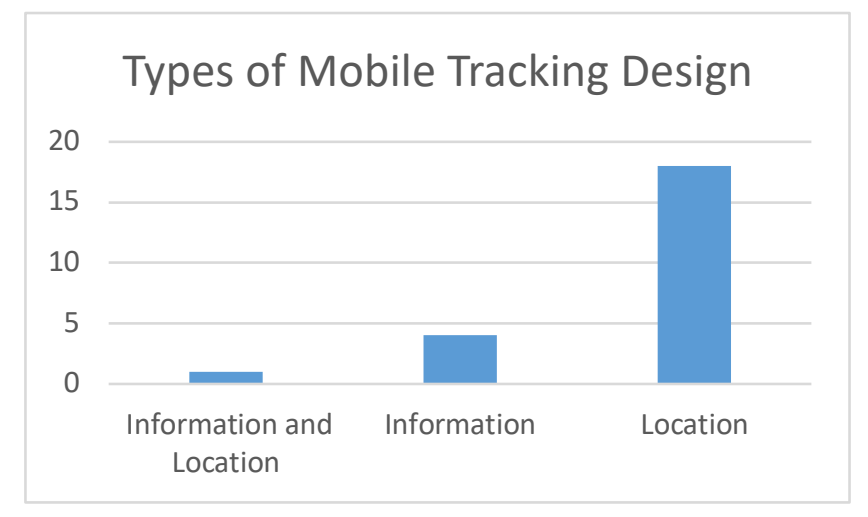

Fig. 4. Results of RQ1

Based on the results of SLR, there are three kinds of the mobile tracking applications, namely: information tracking, location tracking and information and location tracking, as shown in Fig. 4.

As displayed in Fig 6, most of the prior literature utilized the mobile application to determine the location, approximately $78 \%$. In turn, out of 24 studies only $18 \%$ were exploited to information tracking. With regard to the studies designed the application for the information and location tracking was 4\%, Fig. 5 depicted the percentage of these types of the tracking.

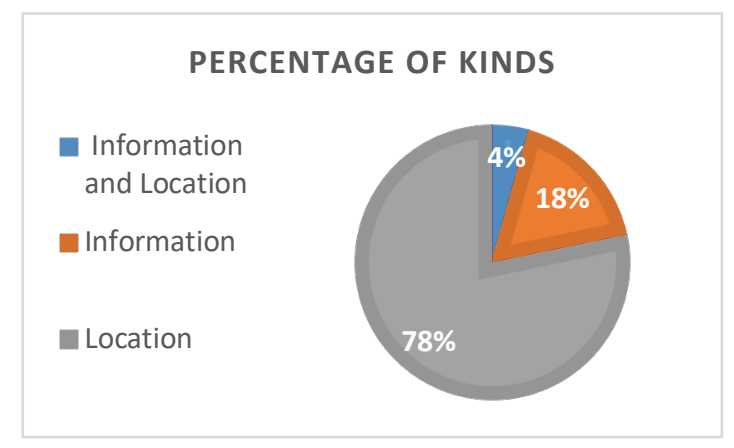

Fig. 5. Percentage of the mobile tracking kinds 
RQ2: How to evaluate the usability of the mobile tracking applications?

In the second research question, the researchers sought to explore what the evaluation techniques exploited to assess the usability of mobile tracking in the previous studies.

\section{USABILITY APPROACHES}

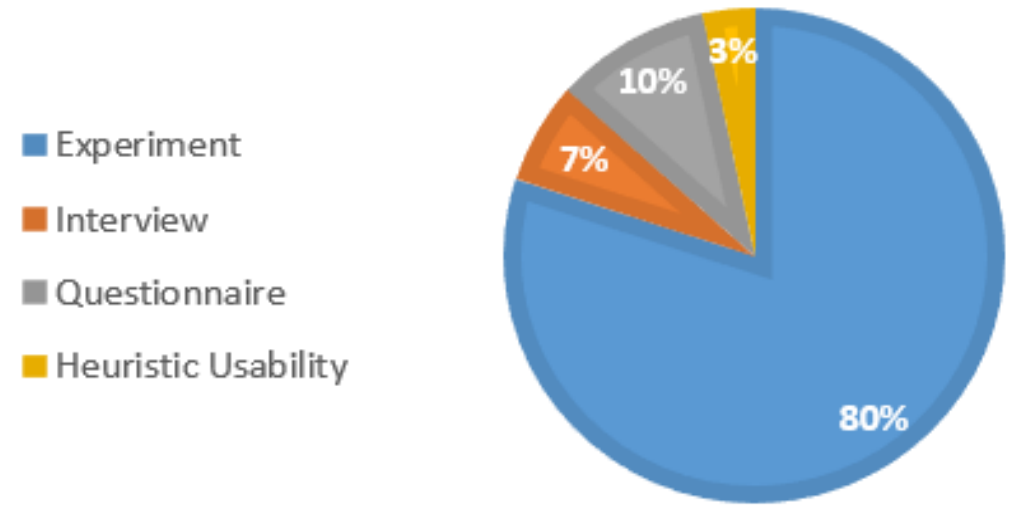

Fig. 6. Results of RQ2

Fig. 6 indicates that, experiment testing was the most technique used by the previous papers to evaluate the usability aspect of the mobile tracking application, roughly $80 \%$ of the previous studies harnessed this approach.

RQ3: What are the usability evaluation characteristics used by prior studies?

To highlight characteristics used through the assessment stage, this systematic review placed this question.

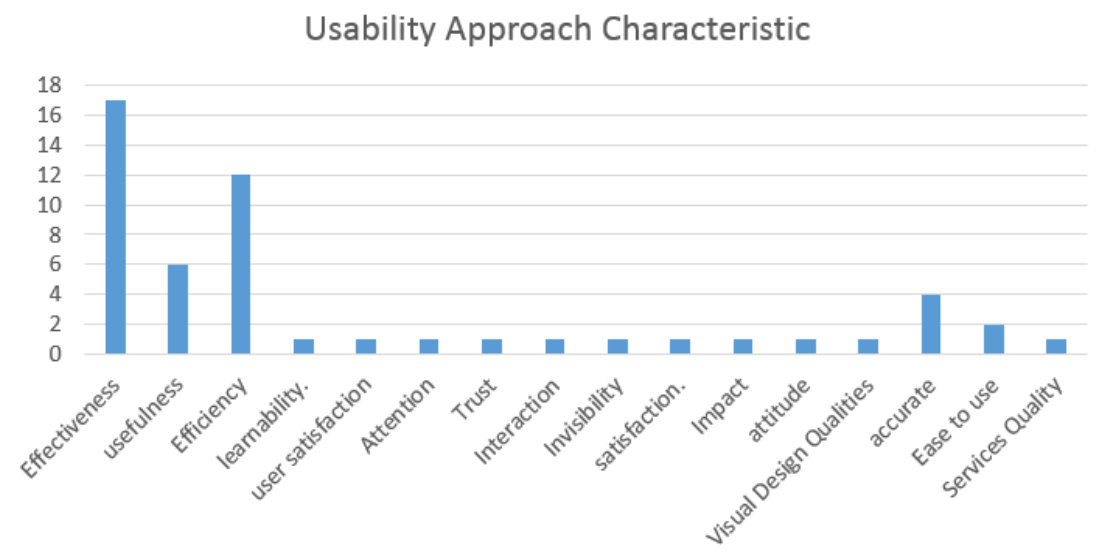

Fig. 7. Characteristics of the evaluation 
Many characteristics were exploited by the previous studies to assess the usability of tracking applications (whether information or location tracking). Three of the popular dimensions of usability assessment were highly used. These dimensions or characteristics were Effectiveness, Usefulness and Efficiency. Thereafter, Accuracy and Ease to use were frequently used.

\section{Conclusion and Future Work}

Usability is considered to be one of the most important quality factors for new technologies (such as web 2.0 and mobile applications), along with others such as reliability and security. More precisely, usability evaluation is more crucial for any website and application.

With regard to this systematic review, three research questions were formulated to dealing with the motivation of present study. The first research question was placed to explore the kinds of the mobile tracking applications. The results of this question indicated that, most of the mobile tracking applications were designed for determine the location (location of vehicle, people or phone). While, other applications were designed for tracking the information.

In the third research question, several techniques were used to measure the usability of the application. The most frequently utilized was experimental study. As for the last research question "What are the usability evaluation characteristics used by prior studies?" the results shown that, four of the characteristics were regularly exploited by the previous studies, these characteristics were: Effectiveness, Efficiency, Usefulness and Accurate.

In the future work directions, the researchers suggested to conduct more systematic review study of the mobile application in different disciplines because there is scarcity of this kind of the study and in the same time there are many applications know designed without concentrates on the usability of the application.

\section{$5 \quad$ References}

[1] S. P. P. Kumar, E. B. Raj, and M. C. P. Selvan, "A Quality Based Power-Hump Mechanism for High Speed Network Connectivity of Mobile Devices in Digital Living Network Alliance Networks," 2015.

[2] L. U. H. Munoz and S. I. Woolley, "A user-centered mobile health device to manage lifethreatening anaphylactic allergies and provide support in allergic reactions," in 2009 9th International Conference on Information Technology and Applications in Biomedicine, 2009, pp. 1-4. https://doi.org/10.1109/itab.2009.5394347

[3] H. Jung, "Cisco visual networking index: global mobile data traffic forecast update 2010 2015,” Technical Report, Cisco Systems Inc. 2011. Available online: https://www ..., 2011.

[4] I. Y. Adam, C. Varol, and A. Varol, "Problems and Prospects of Anti-Theft and Mobile Phone Tracking: A case in Nigeria," in 2019 7th International Symposium on Digital Forensics and Security (ISDFS), 2019, pp. 1-5. https://doi.org/10.1109/isdfs.2019.8757527 
[5] D. Lobo, K. Kaskaloglu, C. Y. Kim, and S. Herbert, "Web usability guidelines for smartphones: a synergic approach,” Int. J. Inf. Electron. Eng., vol. 1, no. 1, pp. 33-37, 2011.

[6] J. Rivera and R. van der Meulen, "Gartner says annual smartphone sales surpassed sales of feature phones for the first time in 2013," Gart. Newsroom, 2014.

[7] M. A. Saare, A. Hussain, and W. S. Yue, "Conceptualizing mobile health application use intention and adoption among Iraqian older adults: from the perspective of expanded technology acceptance model," Int. J. Interact. Mob. Technol., vol. 13, no. 10, pp. 28-41, 2019. https://doi.org/10.3991/ijim.v13i10.11285

[8] B. Liu, J. Lin, and N. Sadeh, "Reconciling mobile app privacy and usability on smartphones: Could user privacy profiles help?" in Proceedings of the 23rd international conference on World Wide Web, 2014, pp. 201-212. https://doi.org/10.1145/2566486. 2568035

[9] F. F.-H. Nah, K. Siau, and H. Sheng, "The value of mobile applications: a utility company study," Commun. ACM, vol. 48, no. 2, pp. 85-90, 2005. https://doi.org/10.1145/1042091. $\underline{1042095}$

[10] C. K. Coursaris and D. J. Kim, "A meta-analytical review of empirical mobile usability studies,” J. usability Stud., vol. 6, no. 3, pp. 117-171, 2011.

[11] M. A. Saare, A. Hussain, and W. S. Yue, "Relationships between the Older Adult's Cognitive Decline and Quality of Life: The Mediating Role of the Assistive Mobile Health Applications," Int. J. Interact. Mob. Technol., vol. 13, no. 10, pp. 42-55, 2019. https://doi. org/10.3991/ijim.v13i10.11288

[12] M. Saare, A. Hussain, and W. S. Yue, "Investigating the Effectiveness of Mobile Peer Support to Enhance the Quality of Life of Older Adults: A Systematic Literature Review," 2019. https://doi.org/10.3991/ijim.v13i04.10525

[13] J. Clarke, "What is a systematic review?" Evid. Based. Nurs., vol. 14, no. 3, p. 64, 2011.

[14] B. Kitchenham, "Procedures for performing systematic reviews," Keele, UK, Keele Univ., vol. 33, no. 2004, pp. 1-26, 2004.

[15] C. Salvador, A. Nakasone, and J. A. Pow-Sang, "A systematic review of usability techniques in agile methodologies," in Proceedings of the 7th Euro American Conference on Telematics and Information Systems, 2014, p. 17. https://doi.org/10.1145/2590651.2590 668

[16] E. Turban, J. Outland, D. King, J. K. Lee, T.-P. Liang, and D. C. Turban, "Mobile commerce and the internet of things," in Electronic Commerce 2018, Springer, 2018, pp. 205248. https://doi.org/10.1007/978-3-319-58715-8 6

[17] E. Turban, J. Whiteside, D. King, and J. Outland, "Mobile commerce and the internet of things," in Introduction to Electronic Commerce and Social Commerce, Springer, 2017, pp. 167-199.

[18] B. Kitchenham and S. Charters, "Guidelines for performing systematic literature reviews in software engineering," 2007 https://doi.org/10.1007/978-3-319-50091-1_6

\section{Authors}

Murtaja Ali Saare is a Ph.D. student at School of Computing, Sintok, Universiti Utara Malaysia, Kedah, Malaysia. He holds a master's degree in computer science. His research interests include aging and cognition, e-health, and human-computer interaction. He has published his work in reputable Scopus indexed journals. saareumm10@gmail.com 
Azham Bin Hussain is the Associate Professor of Software Engineering at UUM School of Computing. He is the founder and head of Human-Centered Computing Research Group, which is affiliated with the Software Technology Research Platform Center at School of Computing, Universiti Utara Malaysia. Assoc. Prof. Azham Hussain is a member of the US-based Institute of Electrical and Electronic Engineers (IEEE), and actively involved in both IEEE Communications and IEEE Computer societies. Azham is published in the areas of software evaluation and testing, user behaviours, group collaboration, and ubiquitous and mobile technology design. He has authored and co-authored more than 100-refereed technical publications, served as reviewer and referee for refereed journals and conferences on computing as well as the examiner for more than twenty doctoral and postgraduate scholars in his research areas. azham.h@uum.edu.my

Osamah Mohammed Jasim is a Master Student in Information Technology at School of Computing, Universiti Utara Malaysia. His research focus on Human Computer Interaction and User Experience. osamahmohammed247@gmail.com

Alia Ahmed Mahdi is working at Office of the Inspector General, Directorate of Health, Iraq, has completed her master's in information technology and looking forward to further to $\mathrm{PhD}$ in Human Computer Interaction or Software Requirement for mobile. alia ahmed m@gmail.com

Article submitted 2019-12-17. Resubmitted 2020-01-08. Final acceptance 2020-01-10. Final version published as submitted by the authors. 